Version in english:

http://www.conhecer.org.br/enciclop/2020C/perfil2.pdf
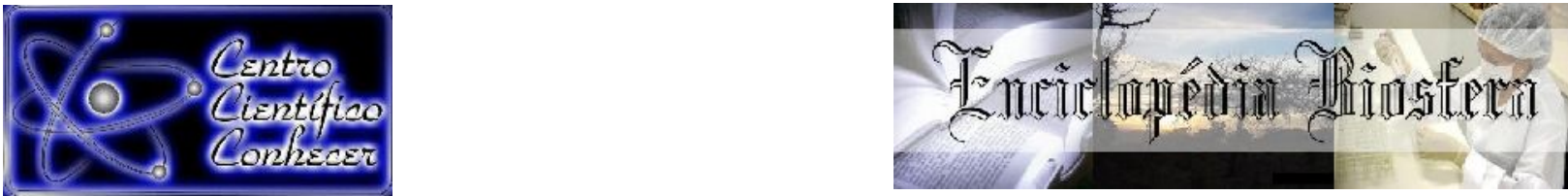

\title{
PERFIL DE ANSIEDADE EM AGENTES DE UM PRESÍDIO: ESTUDO DE CASO
}

\section{Jean da Costa Silva ${ }^{1}$, Amanda Melo Sant'Anna Araújo², Eric Francelino Andrade ${ }^{3}$,} Débora Ribeiro Orlando ${ }^{4}$

${ }^{1}$ Graduando em Medicina Veterinária, Universidade Federal dos Vales do Jequitinhonha e Mucuri (UFVJM) - Instituto de Ciências Agrárias Unaí, MG - Brasil.

${ }^{2}$ Médica Veterinária, Profa. Mestre, Universidade Federal dos Vales do Jequitinhonha e Mucuri (UFVJM) - Instituto de Ciências Agrárias, Unaí, MG - Brasil.

${ }^{3}$ Educador Físico, Prof. Doutor, Universidade Federal dos Vales do Jequitinhonha e Mucuri (UFVJM) - Instituto de Ciências Agrárias, Unaí, MG - Brasil.

${ }^{4}$ Médica Veterinária, Profa. Doutora, Universidade Federal dos Vales do Jequitinhonha e Mucuri (UFVJM) - Instituto de Ciências Agrárias/Campus Unaí-MG

Avenida Universitária, 1000, Universitários, Unaí, MG - Brasil (deboraribeiro.orlando@gmail.com)

Recebido em: 15/08/2020 - Aprovado em: 15/09/2020 - Publicado em: 30/09/2020 DOI: 10.18677/EnciBio_2020C30

\begin{abstract}
RESUMO
Algumas profissões apresentam potenciais agentes estressores que podem desencadear transtornos psicológicos como a ansiedade. Neste sentido, profissionais de segurança podem ser altamente susceptíveis a desenvolver quadros de ansiedade devido às características presentes em seu setor laboral. Assim, objetivou-se com este estudo, avaliar o perfil de ansiedade em agentes penitenciários trabalhadores de um presídio situado no noroeste do estado de Minas Gerais. A amostra foi composta por 47 servidores (oito mulheres e 39 homens) com idade entre 25 e 53 anos. Para avaliação e classificação dos níveis de ansiedade, os participantes responderam ao Inventário de Ansiedade Traço-Estado (IDATE). Os dados foram submetidos à estatística descritiva, análise de variância (ANOVA) e teste do qui-quadrado e as médias comparadas pelo teste de t de student $(p<0,05)$. Observou-se uma maior frequência de indivíduos com baixo nível de ansiedade $(p<$ 0,05), sendo que aqueles que relataram uso de medicamentos psicotrópicos apresentaram maiores escores de ansiedade estado $(p<0,05)$. Não foi observada diferença significativa nos escores de ansiedade na comparação entre gêneros e com relação ao convívio com animais de estimação. Concluiu-se que, na amostra avaliada de agentes penitenciários, houve maior prevalência de ansiedade em nível baixo, sendo o uso de fármacos psicotrópicos associado a níveis mais elevados deste transtorno.
\end{abstract}

PALAVRAS-CHAVE: Ansiedade. Profissionais de segurança. Penitênciária. 


\title{
PROFILE OF ANXIETY IN PENITENTIARY AGENTS
}

\begin{abstract}
Some professions have potential stressors that can trigger psychological disorders such as anxiety. In this sense, security professionals may be highly susceptible to developing anxiety disorders due to the characteristics of their work segment. Thus, the aim of our study was to evaluate the profile of anxiety among penitentiary agents that work in a prison located in the northwest of the state of Minas Gerais, Brazil. The sample consisted of 47 workers ( 8 women and 39 men) aged between 25 and 53 years. To assess and classify anxiety levels, participants responded to the State-Trait Anxiety Inventory (STAI). The data were submitted to descriptive statistics, analysis of variance (ANOVA) and chi-square test and the means compared by the student's t test $(p<0.05)$. There was a higher frequency of individuals with low anxiety levels ( $p$ $<0.05$ ) and those who reported using psychotropic treatments had higher scores of state anxiety $(p<0.05)$. There was no significant difference in anxiety scores when comparing genders and in relation to living with pets. We concluded that, in the sample evaluated of penitentiary agents, there is a higher prevalence of anxiety at a low level, with the use of psychotropic drugs associated with higher levels of this disorder.
\end{abstract}

KEYWORDS: Anxiety. Security professionals. Penitentiary.

\section{INTRODUÇÃO}

A ansiedade é um estado emocional resultante de sentimentos atípicos de tensão, apreensão, preocupação, inquietação e medo acerca de situações incertas ou que ainda não aconteceram (SHAPIRO et al., 2014). Assim, diante de um desafio ou uma situação estressante, o organismo desenvolve estratégias de enfrentamento (coping) para se adaptar e, quando este processo não é eficaz, ocorrem manifestações psicossomáticas que prejudicam o bem-estar físico e mental (FLECK, 2000). A ansiedade pode ser de origem situacional ou da personalidade do indivíduo, podendo, em ambos os casos, interferir de forma negativa na concentração e nas formas de solucionar problemas comuns do dia a dia (CHAVES; CADE, 2002).

Levando em consideração estas características da ansiedade, o Inventário de Ansiedade Traço-Estado (IDATE) tem sido considerado um dos mais importantes instrumentos usados para avaliar, quantitativamente, os componentes considerados subjetivos sobre esta condição (BIAGGIO et al., 1977). Desenvolvido por Spielberger et al. (1970) e traduzido para o português por Biaggio et al. (1977), esse instrumento apresenta uma escala que permite avaliar a ansiedade nas dimensões estado (IDATE-E) e traço (IDATE-T). A ansiedade-estado é uma condição emocional transitória caracterizada por sentimentos desagradáveis de tensão e apreensão conscientes que são percebidos causando aumento da atividade do Sistema Nervoso Autônomo Simpático (LEAL et al., 2017). Já ansiedade traço está relacionada às características particulares de um indivíduo, ou seja, nas reações deste às situações percebidas como ameaçadoras, que eleva as intensidades no estado de ansiedade (BIAGGIO et al., 1977).

Algumas profissões podem ser mais propensas à ansiedade que outras (ANDRADE et al., 2020). Nesse sentido, agentes penitenciários são trabalhadores importantes para o funcionamento das instituições prisionais que, frequentemente, ENCICLOPÉDIA BIOSFERA, Centro Científico Conhecer - Jandaia-GO, v.17 n.33; p. 340 2020 
estão sujeitos a situações que geram ansiedade pois trabalham sob pressão, estão sujeitos a risco de morte e recebem pouco reconhecimento da sociedade (BEZERRA et al., 2016). Além disso, considera-se que este grupo profissional é pouco avaliado em estudos devido à dificuldade de acesso por parte das instituições prisionais (BEZERRA et al., 2016).

Neste sentido, considerando que agentes penitenciários estão sujeitos a diversos fatores que podem desencadear ansiedade, objetivou-se com o presente estudo, avaliar o perfil de ansiedade em agentes penitenciários de um presídio do noroeste de Minas Gerais.

\section{Participantes}

\section{MATERIAIS E MÉTODOS}

Participaram do estudo 47 agentes penitenciários (oito mulheres e 39 homens) com idade entre 25 e 53 anos, servidores da Penitenciária Agostinho de Oliveira Júnior da cidade de Unaí, Minas Gerais. A amostra compreendeu apenas os servidores que estavam em serviço durante o mês de outubro de 2019, que foi o período de coleta dos dados. No momento da coleta de dados a população carcerária era de 688 indivíduos. Após preenchimento do Termo de Consentimento Livre e Esclarecido (TCLE), os agentes responderam, voluntariamente, ao Inventário de Ansiedade Traço-Estado (IDATE) (SPIELBERGER et al., 1970).

\section{Instrumento}

O IDATE desenvolvido por Spielberger et al. (1970) permite avaliar a ansiedade nas dimensões estado (IDATE-E) e traço (IDATE-T). Este inventário apresenta 40 questões (20 relacionadas ao IDATE-E e outras 20 ao IDATE-T) com possibilidade de resposta em escala Likert de quatro pontos ("muitíssimo", "bastante", "um pouco", e "absolutamente não"). Os níveis de ansiedade, tanto estado quanto traço, foram obtidos através da soma dos valores obtidos após a conversão das respostas em números. Assim, quanto maior o resultado calculado, maior o nível de ansiedade apresentado pelo respondente. Desta forma, para cada dimensão do IDATE existe uma possibilidade de resultados que variam de um mínimo de 20 pontos até ao máximo de 80 pontos (GERRETH et al., 2019). Adicionalmente, foram atribuídas classificações quanto ao nível de ansiedade, sendo que resultados entre 20 e 40 foram classificados como "baixo", entre 41 e 60 "moderado" e entre 61 e 80 pontos como "alto" nível de ansiedade (GERRETH et al., 2019). Ao final do IDATE foram inseridas questões sobre aspectos como gênero, convívio com animais de estimação e uso de medicamentos psicotrópicos.

\section{Análises Estatísticas}

Os dados foram submetidos à estatística descritiva e Análise de Variância (ANOVA) e as médias comparadas por teste t de student. A frequência de indivíduos situadas em cada nível de ansiedade foi comparada pelo teste do qui-quadrado. Foram consideradas diferenças significativas um nível de probabilidade menor que $5 \%(p<0,05)$. Os dados foram analisados através do software estatístico BioEstat 5.3 (AYRES et al., 2007). 


\section{RESULTADOS}

Foi observada menor prevalência de indivíduos classificados com nível da ansiedade alto em comparação aos classificados como baixo e moderado no IDATE$E$, IDATE-T e na ansiedade total ( $p<0,05$ - Figura 1).

FIGURA 1 - Prevalência de indivíduos classificados com diferentes níveis de ansiedade.

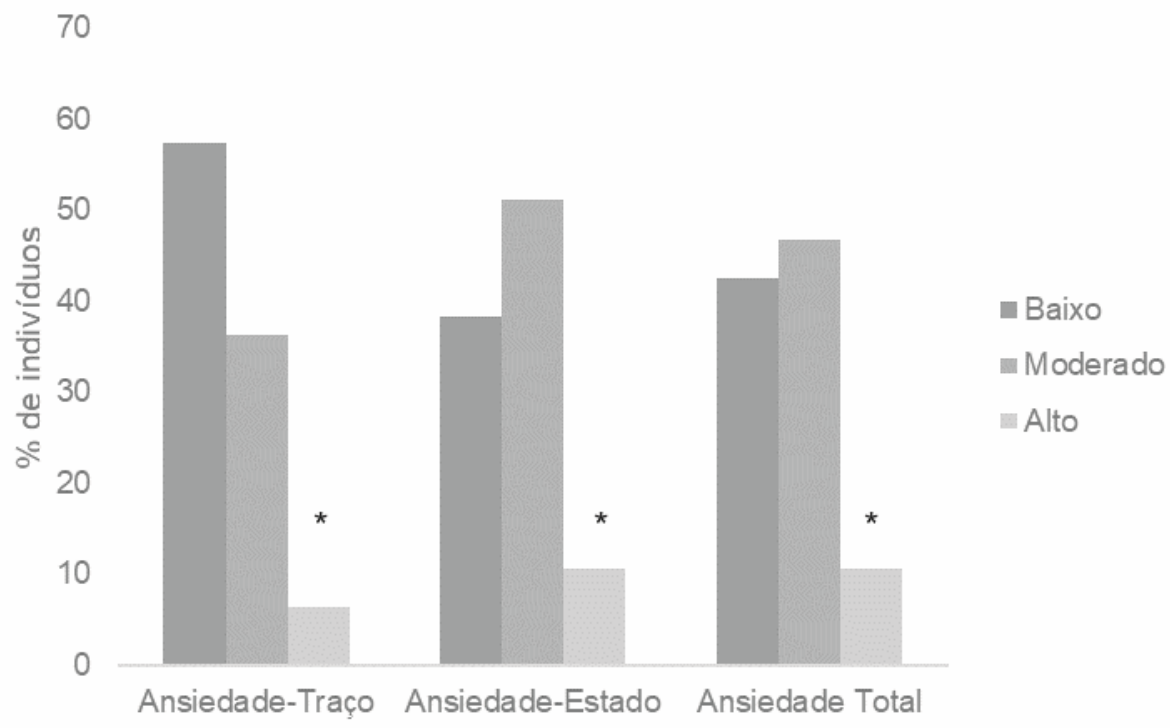

* Menores níveis de ansiedade.

Com relação aos escores de ansiedade, não houve diferenças significativas na comparação entre gênero e entre convívio com animal de estimação para nenhuma das classificações de ansiedade (Tabela 1). Os escores do IDATE-T foram maiores nos participantes que relataram uso de medicamentos psicotrópicos em comparação aos que não usam $(p<0,05)$. Todavia, não foram observadas diferenças para este aspecto no IDATE-E e no escore total de ansiedade (Tabela 1). 
TABELA 1 - Escores de ansiedade em agentes penitenciários.

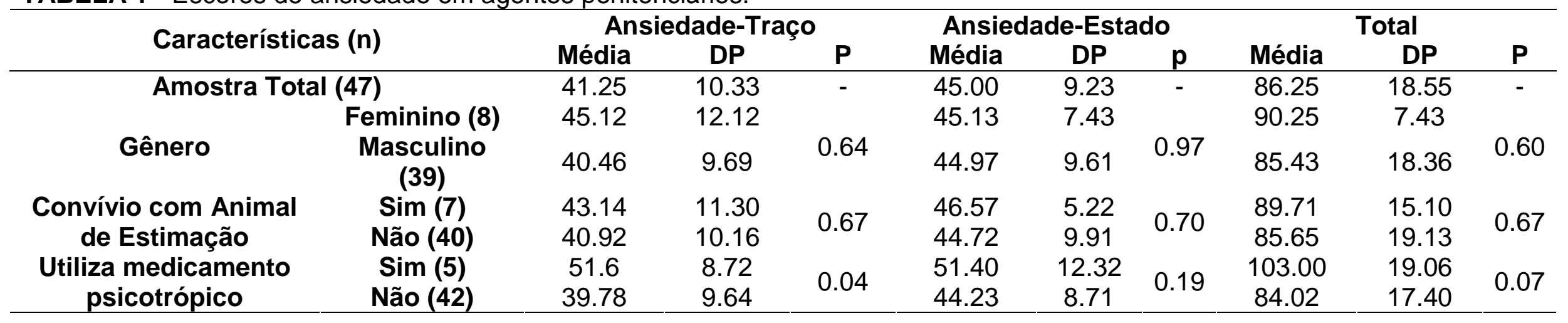




\section{DISCUSSÃO}

No presente estudo foi observada maior prevalência de agentes penitenciários classificados com ansiedade leve. Estes resultados são surpreendentes, já que esta classe de trabalhadores vivencia, frequentemente, situações estressantes que podem gerar ansiedade (BEZERRA et al., 2016; BONEZ et al. 2017). Diversos estudos relatam níveis elevados de ansiedade neste setor de trabalho (BEZERRA et al., 2016; LIMA et al., 2019; REINERT et al. 2019). Foram observadas altas intensidades de ansiedade em agentes de uma penitenciária do Estado do Mato Grosso antes de serem submetidos a três semanas de auriculoterapia (GRAÇA et al., 2020). Entretanto, corroborando os resultados encontrados no presente estudo, Bonez et al. (2017) observaram que a maioria dos agentes de um presídio do estado de Santa Catarina apresentaram baixo nível de ansiedade e nenhum grau de depressão e desesperança. Mesmo assim, os autores supracitados alertam sobre o cuidado com a saúde mental nesta classe de trabalhadores, já que os níveis de ansiedade e estresse podem aumentar de forma rápida.

Agentes penitenciários são submetidos diariamente a situações de desconforto, pressão psicológica e sobrecarga laboral que acarretam sintomas psicossomáticos (BONEZ et al., 2017). Além disso, considera-se que o ambiente de trabalho provoca medo de que ocorram lesões físicas, deixando estes indivíduos frequentemente alertas e vigilantes dentro e fora do ambiente de trabalho (LIMA et al., 2019).

A maior prevalência de agentes com baixo nível de ansiedade observada foi decorrente de alguma variável ou estratégia de coping não investigada no presente estudo. Este argumento pode ser reforçado pela discrepância observada, no presente estudo, entre indivíduos que utilizam fármacos psicotrópicos em comparação aos que não usam. A literatura relata consumo elevado destes medicamentos em servidores da área de segurança (FIGUEIRÓ et al., 2015; BADRU et al., 2018). Em estudo realizado com oficiais correcionais de uma prisão Nigeriana foi observada uma relação diretamente proporcional entre o uso de psicofármacos a presença de ansiedade e depressão (BADRU et al., 2018). Estes resultados corroboram com os observados no presente estudo para o IDATE-T, onde foram verificados maiores escores de ansiedade traço nos agentes que utilizam medicamentos psicotrópicos.

Com relação ao gênero, no presente estudo não houve diferença entre os escores de ansiedade entre homens e mulheres. Este é outro resultado intrigante do presente estudo, já que a literatura relata que a ansiedade acomete mais o sexo feminino que o masculino (STEIMER, 2011; ANDRADE et al., 2018; ANDRADE et al., 2020). Adicionalmente, foi relatado que quando se trata de agentes penitenciários, as mulheres apresentam maiores níveis de ansiedade que os homens (SILVA et al., 2017).

No presente estudo também foi investigado se o convívio com animal de estimação influencia os níveis de ansiedade, e não foi observada diferença significativa. Em estudo prévio (ANDRADE et al., 2018) foi observado que estudantes universitários que convivem com animais de estimação apresentam menores níveis de ansiedade no IDATE-E. Esta discrepância pode ser explicada pelo fato de que a presença de pet pode atenuar a ansiedade em grupos que apresentam alta prevalência de ansiedade em níveis elevados, como foi o caso de estudantes universitários (ANDRADE et al., 2018). 
Considerando que o IDATE é um instrumento psicométrico que permite observar apenas resultados quantitativos, é importante que estudos futuros avaliem aspectos qualitativos, por exemplo, através de análise do discurso nesta população. Macedo et al. (2008) relatam que a análise do discurso em estudos na área da saúde permite a interpretação da realidade e a identificação de contradições com os resultados quantitativos.

\section{AGRADECIMENTOS}

Ao Conselho Nacional de Desenvolvimento Científico e Tecnológico (CNPq) pela concessão da bolsa de iniciação científica e à Universidade Federal dos Vales do Jequitinhonha e Mucuri (UFVJM).

\section{REFERÊNCIAS}

ANDRADE, K. A.; ARAÚJO, A. M. S.; ANDRADE, E. F.; ORLANDO, D. R. University Students that Living with Pets Present Lower Levels of Anxiety. Enciclopédia Biosfera, v. 15, n. 28, p. 896-906, 2018. Disponível em: <http://www.conhecer.org.br/enciclop/2018B/AGRAR/universitarios.pdf>. $\quad$ doi: 10.18677/EnciBio_2018B73

ANDRADE, K. A.; ARAÚJO, A. M. S.; ANDRADE, E. F.; ORLANDO, D. R. Perfil de ansiedade em servidores de uma instituição de ensino superior do noroeste de Minas Gerais. Enciclopédia Biosfera, v. 17, n. 32, p. 422-430, 2020. Disponível em: <http://www.conhecer.org.br/enciclop/2020B/perfil\%20de.pdf>. doi: 10.18677/EnciBio_2020B38

AYRES, M.; AYRES JÚNIOR, M.; AYRES, D. L. E SANTOS, A. A. BIOESTAT Aplicações estatísticas nas áreas das Ciências Bio-Médicas. Belém:Sociedade Civil Mamirauá; 2007.

BADRU, O. A.; OGUNLESI, A. O.; OGUNWALE, A.; ABDULMALIK, J. O.; YUSUF, $O$. B. Prevalence of generalized anxiety disorder and major depression among correctional officers in a Nigerian prison. Journal of Forensic Psychiatry and Psychology, v. 29, n. 4, p. 509-526, 2018. Routledge. Disponível em: <https://www.tandfonline.com/doi/abs/10.1080/14789949.2017.1421250>. doi: 10.1080/14789949.2017.1421250

BEZERRA, C. DE M.; DE ASSIS, S. G.; CONSTANTINO, P. Sofrimento psíquico e estresse no trabalho de agentes penitenciários: Uma revisão da literatura. Ciência e Saúde Coletiva, v. 21, n. 7, p. 2135-2146, 2016. Disponível em: <https://www.scielo.br/scielo.php?pid=S141381232016000702135\&script=sci_abstra ct\&tlng=pt>. doi: 10.1590/1413-81232015217.00502016

BIAGGIO, A. M. B.; NATALÍCIO, L.; SPIELBERGER, C. D. Desenvolvimento da forma experimental em português do Inventário de Ansiedade Traço-Estado (IDATE) de Spielberger. Arquivos Brasileiros de Psicologia Aplicada, v. 29, n. 3, p. 31-44, 1977. O'Reilly. Disponível em: <http://bibliotecadigital.fgv.br/ojs/index.php/abpa/article/view/17827>.

BONEZ, A.; DAL MORO, E.; SEHNEM, S. B. Saúde mental de agentes ENCICLOPÉDIA BIOSFERA, Centro Científico Conhecer - Jandaia-GO, v.17 n.33; p. 345 
penitenciários de um presídio catarinense. Psicologia Argumento, v. 31, n. 74, p. 507-517, $2017 . \quad$ Disponível em: <https://periodicos.pucpr.br/index.php/psicologiaargumento/article/view/20585>. doi: 10.7213/psicol.argum.31.074.AO05

CHAVES, E. C.; CADE, N. V. Enfrentamento e sua relação com a ansiedade e com a depressão em mulheres com hipertensão. Revista Brasileira de Terapia Comportamental e Cognitiva, v. 4, n. 1, p. 1-11, 2002. Disponível em: $<$ http://www.usp.br/rbtcc/index.php/RBTCC/article/view/118>. doi.org/10.31505/rbtcc.v4i1.118

FIGUEIRÓ, R. A.; DIMENSTEIN, M.; ALVES, D.; MEDEIROS, G. Consumo de medicação psicotrópica em uma prisão feminina. Revista Psicologia Política, v. 15, n. $34, \quad$ p. 531-547, 2015. Disponível em: <http://pepsic.bvsalud.org/scielo.php?script=sci_arttext\&pid=S1519549X2015000300006>.

FLECK, M. P. DE A. O instrumento de avaliação de qualidade de vida da Organização Mundial da Saúde (WHOQOL-100): características e perspectivas. Ciência \& Saúde Coletiva, v. 5, n. 1, p. 33-38, 2000. Associação Brasileira de Saúde Coletiva. Disponível em: <http://www.scielo.br/scielo.php?script=sci_arttext\&pid=S1413-

$81232000000100004 \&$ Ing=pt\&tlng=pt>. doi: $10.1590 /$ S1413-81232000000100004

GERRETH, K.; CHLAPOWSKA, J.; LEWICKA-PANCZAK, K.; SNIATALA, R.; EKKERT, M.; et al. Self-Evaluation of Anxiety in Dental Students. BioMed Research International, v. 2019, p. 6436750, 2019. Disponível em: <https://www.hindawi.com/journals/bmri/2019/6436750/>. $10.1155 / 2019 / 6436750$

GRAÇA, B. C.; NASCIMENTO, V. F.; FELIPE, R. N. R.; ANDRADE, A. C. S.; ATANAKA, M.; et al. Uso da auriculoterapia no controle da lombalgia, ansiedade e estresse de profissionais do sistema penitenciário. Brazilian Journal of Pain, v. 3, n. 2, p. 142-146, 2020. Disponível em: < https://www.scielo.br/pdf/brjp/v3n2/pt_18060013-brjp-20200025.pdf>. doi: 10.5935/2595-0118.20200025

LEAL, P. C.; GOES, T. C.; DA SILVA, L. C. F.; TEIXEIRA-SILVA, F. Trait vs. state anxiety in different threatening situations. Trends in Psychiatry and Psychotherapy, v. 39, n. 3, p. 147-157, 2017. Disponível em: $<$ https://www.scielo.br/scielo.php?pid=S2237-

60892017000300147\&script=sci_arttext>. doi: 10.1590/2237-6089-2016-0044

LIMA, A. I. O.; DIMENSTEIN, M.; FIGUEIRÓ, R.; LEITE, J.; DANTAS, C. Prevalência de Transtornos Mentais Comuns e Uso de Álcool e Drogas entre Agentes Penitenciários. Psicologia: Teoria e Pesquisa, v. 35, 2019. Disponível em: <https://dx.doi.org/10.1590/0102.3772e3555>. doi: 10.1590/0102.3772e3555

MACEDO, L. C.; LAROCCA, L. M.; CHAVES, M. M. N.; MAZZA, V. A. Análise do discurso: uma reflexão para pesquisar em saúde. Interface. v.12, n.26, p.649-657, ENCICLOPÉDIA BIOSFERA, Centro Científico Conhecer - Jandaia-GO, v. 17 n. 33; p. 346 2020 
2008. Disponível em: < https://www.scielo.br/scielo.php?pid=S141432832008000300015\&script=sci_abstract\&tlng=pt>. doi: 10.1590/S141432832008000300015

REINERT, F.; VERGARA, L. G. L.; GONTIJO, L. A. Percepção das Condições de Trabalho e Saúde pelos Agentes Penitenciários do Presidio Masculino de Florianópolis/SC. Revista Ação Ergonômica, v. 13, n. 1, 2019. Disponível em: <http://www.abergo.org.br/revista/index.php/ae/article/view/491>.

SHAPIRO, A. L.; CULP, S.; AZULAY CHERTOK, I. R. OSA Symptoms Associated With and Predictive of Anxiety in Middle-Aged Men: Secondary Analysis of NHANES Data. Archives of Psychiatric Nursing, v. 28, n. 3, p. 200-205, 2014. Disponível em: <http://www.ncbi.nlm.nih.gov/pubmed/24856274>. doi: 10.1016/j.apnu.2014.02.002

SILVA, A. L. R. R.; SALES, G. E. R. B.; FONSECA, L. F. M. F.; ASTOLFO, M. H.; MANOEL, P. G.; et al. Síndrome Metabólica e Estresse de Agentes de Segurança Penitenciária. Arquivos de Ciências da Saúde, v. 24, n. 3, p. 35, 2017. Disponível em: <http://www.cienciasdasaude.famerp.br/index.php/racs/article/view/755>. doi: 10.17696/2318-3691.24.3.2017.755

SPIELBERGER, C. D.; GORSUCH, R. L.; LUSHENE, R. E. Manual for the StateTrait Anxiety Inventory. $1^{\circ}$ ed. Palo Alto, CA: Consulting Psychologists Press, 1970. STEIMER, T. Animal models of anxiety disorders in rats and mice: some conceptual issues. Dialogues in clinical neuroscience, v. 13, n. 4, p. 495-506, 2011. Disponível em: <http://www.ncbi.nlm.nih.gov/pubmed/22275854>. 